\title{
Mechanical thrombectomy in unknown time-of-onset stroke: 237 case experience in a tertiary care center.
}

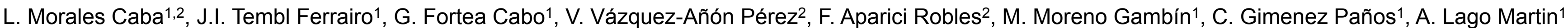
${ }^{1}$ Hospital Universitari i Politècnic La Fe, Neurology Department Stroke Unit, Valencia, Spain.

${ }^{2}$ Hospital Universitari i Politècnic La Fe, Interventional Neuroradiology, Valencia, Spain.

\section{Background.}

New guidelines establish that acute stroke mechanical thrombectomy (MT) can be performed up to 24 hours after last seen normal, providing a significant infarct volume is ruled out with perfusion neuroimaging.

Our objective is to know if the prognosis differs in patients undergoing mechanical thrombectomy (MT) with unknown time of onset (MT_UT) vs known time of onset (MT_KT) in our center.

\section{Method.}

Select all patients treated with MT from January 2014 to July 2018 in Hospital Universitari i Politécnic La Fe with three month after discharge outcome data available.

For both MT_UT and MT_KT groups, demographic characteristics, cardiovascular risk factors, ASPECTS-TC, perfusion mismatch, and outcomes (NIHSS pre MT and at discharge, mRS at discharge and at three months, $\mathrm{mRS} \geq 2$ at three months, and death) were analysed.

\section{Results.}

237 patients were included. 50 were MT_UT -of them 29 being wake up strokes (58\%).

Fibrinolysis treatment was significantly more prevalent among KT patients, as rtPA use in UT stroke patients is not recommended, although it was administered in $21,1 \%$ of our unknown time-of-onset on a radiological basis (low core volume, high mismatch).

MT_KT and MT_UT patients were comparable in terms of age, sex, cardiovascular risk and stroke severity :

- Initial NIHSS: MT_KT: 16.40, MT_UT:17.13, p=0.354.

- ASPECTS-TC: MT_KT 8.45, MT_UT 7.76, $p=0.112$.

- Mismatch: MT_KT 75.93, MT_UT 75.56, p=0.966.

No differences in immediate results were found:

- Discharge NIHSS: MT_KT 8.524, MT_UT 8.815, p=0.838.

- Discharge mRS: MT_KT 3,55, MT_UT 3,53, p=0.53.

And also three months follow up revealed no significant differences in functional outcome:

- Mean mRS: MT_KT 3,26\%, MT_UT 2,97\%, $p=0,51$

- $m R S \geq 2$ : MT_KT 56,2\% vs MT_UT 60,5\%, $p=0,34$.

- Mortality: MT_KT 19,9\%, MT_UT 21,5\%, $p=0,68$.
Conclussions:

* Patients treated in our center with either wake up or unknown time of onset strokes had similar baseline characteristics, clinico-radiological presentation, mortality and functional outcome than strokes treated with a known time of onset.

* Currently used perfusion softwares could sufficiently determine good candidates for MT beyond 6 hours.

\begin{tabular}{|l|c|c|l|}
\hline $\mathbf{n}$ & \multicolumn{1}{|c|}{ Known onset Unknown } & p value \\
\hline Age (range) & $66(15-89)$ & $64(33-82)$ & 0,59 \\
\hline Gender male (\%) & $57 \%$ & $50 \%$ & 0,7 \\
\hline NIHSS admission & 17,12 & 16,40 & 0,35 \\
\hline ASPECTS & 8,45 & 7,76 & 0,112 \\
\hline Core volume (mL) & 39,72 & 51,34 & 0,73 \\
\hline Mismatch (\%) & 75,56 & 75,93 & 0,96 \\
\hline rtPA (\%) & $78,9 \%$ & $21,1 \%$ & $\mathbf{0 , 0 4}$ \\
\hline mRS discharge & 3,55 & 3,53 & 0,53 \\
\hline NIHSS discharge & 8,81 & 8,52 & 0,83 \\
\hline mRS at $\mathbf{3}$ m & 3,26 & 2,97 & 0,51 \\
\hline mRS $\geq \mathbf{2}$ at $\mathbf{3}$ m & $56,2 \%$ & $60,5 \%$ & 0,34 \\
\hline mRS = 6 at $\mathbf{3} \mathbf{m}$ & $19,9 \%$ & $21,5 \%$ & 0,68 \\
\hline
\end{tabular}

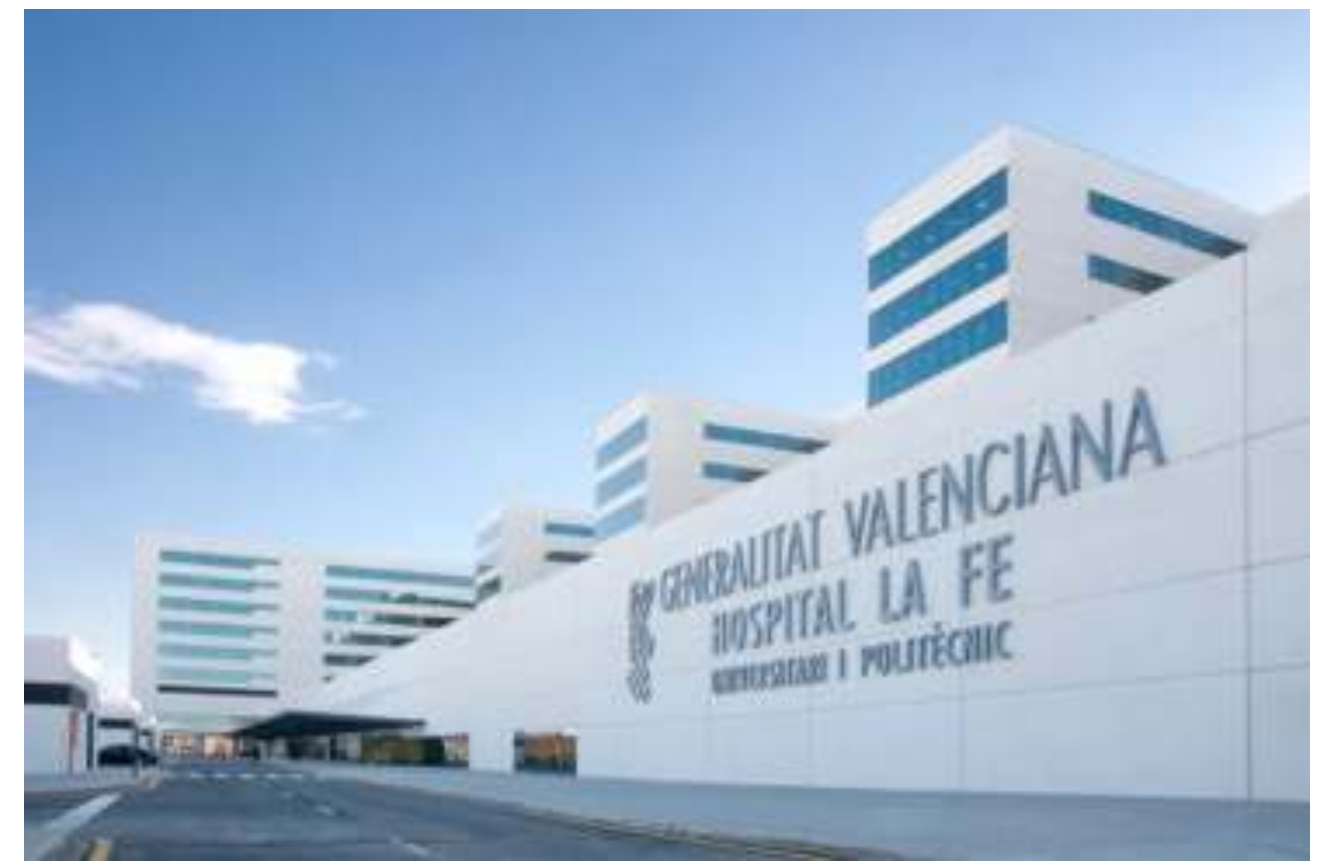

